\title{
Trastuzumab Treatment beyond Progression in Advanced Breast Cancer: Patterns of Care in Six Swiss Breast Cancer Centers
}

\author{
Jens Huober ${ }^{\mathrm{a}}$ Michael Baumann ${ }^{\mathrm{a}}$ Christoph Rochlitz ${ }^{\mathrm{b}}$ Stefan Aebi ${ }^{\mathrm{e}}$ \\ Uwe Güth $^{c}$ Roger von Moos ${ }^{f}$ Andreas Müllerg Lukas von Rohr ${ }^{h}$ \\ Isabelle Widmerd Beat Thürlimann ${ }^{\mathrm{a}}$ \\ ${ }^{a}$ Breast Center, Kantonsspital St. Gallen, St. Gallen, Departments of ${ }^{b}$ Medical Oncology and ${ }^{\mathrm{C}}$ Gynecology, \\ Universitätsfrauenspital, and d F. Hoffmann-La Roche Ltd., Basel, e Departments of Medical Oncology and \\ Gynecology, Breast and Gynecological Cancer Center, Bern, ${ }^{f}$ Department of Medical Oncology, Kantonsspital \\ Graubünden, Chur, ${ }^{9}$ Medical Oncology, Department of Internal Medicine, Kantonsspital Winterthur, Winterthur \\ and hepartment of Oncology, Kantonsspital Aarau, Aarau, Switzerland
}

\section{Key Words}

Brain metastases $\cdot$ Chemotherapy $\cdot$ Clinical practice $\cdot$

HER2 - Lapatinib • Metastatic breast cancer · Trastuzumab

\begin{abstract}
Background: Trastuzumab is an established treatment for HER2-positive breast cancer (BC). We analyzed Swiss patterns of care in patients with HER2-positive BC after disease progression on trastuzumab-containing therapy for metastatic $\mathrm{BC}$ (MBC). Patients and Methods: A retrospective analysis was performed in six Swiss BC centers. Patients with HER2-positive MBC treated with at least one infusion of trastuzumab for advanced disease between January 2006 and December 2007 were identified. Treatment patterns in first and further lines were analyzed. Results: All of the 72 identified patients received trastuzumab as their first palliative anti-HER2 therapy, either as monotherapy $(n=23)$ or in combination with chemotherapy (typically taxane or vinorelbine; $n=49$ ). Median time to progression was 8.1, 8.0 and 7.9 months in the monotherapy, trastuzumab-taxane and trastuzumab-vinorelbine cohorts, respectively. After progression on first-line anti-HER2 therapy, trastuzumab was continued in 67 of 68 patients who received further therapy.
\end{abstract}

One patient received second-line lapatinib plus capecitabine. The median duration of anti-HER2 therapy was 20 months. Patients received a median of 4 lines of anti-HER2 therapy. Conclusions: Durable responses were achieved with repeated exposure to anti-HER2 therapy. In a selected patient population, trastuzumab monotherapy appears to be a reasonable first-line treatment option.

Copyright $\odot 2011$ S. Karger AG, Basel

\section{Introduction}

Trastuzumab-based treatment is the standard of care for patients with human epidermal growth factor receptor 2 (HER2)-positive breast cancer. Trastuzumab has been shown to significantly improve outcomes, including overall survival, in randomized phase III trials in both early and metastatic breast cancer. In HER2-overexpressing metastatic breast cancer (MBC), Slamon et al. [1] demonstrated significantly improved time to disease pro-

J.H. and M.B. contributed equally to this work.

\section{KARGER}

Fax +41613061234 E-Mail karger@karger.ch www.karger.com

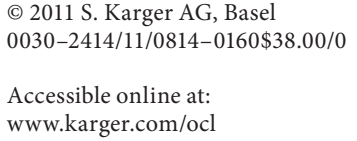

\section{Jens Huober, MD}

Breast Center

Kantonsspital St. Gallen

CH-9007 St. Gallen (Switzerland)

Tel. +41 71494 1880,E-Mail jens.huober@ kssg.ch 
gression (TTP; primary endpoint), response rate and overall survival in patients randomized to trastuzumab in combination with chemotherapy compared with patients receiving chemotherapy alone. This observation was confirmed in the M77001 trial evaluating docetaxel with or without trastuzumab as first-line therapy for MBC [2]. More recently, the TAnDEM (Trastuzumab and Anastrozole Directed Against ER-Positive HER2-Positive Mammary Carcinoma) trial showed that the addition of trastuzumab to anastrozole in postmenopausal women with HER2-positive hormone-receptor-positive MBC significantly improved progression-free survival (PFS) and the clinical benefit rate [3]. A phase II study showed that trastuzumab is active as monotherapy in the first-line treatment of HER2-positive MBC, with response rates of up to $35 \%$ and a median TTP of almost 4 months [4].

Unfortunately, most patients will eventually experience disease progression, posing a therapeutic challenge. Lapatinib in combination with capecitabine is an approved option in this setting, based on results from the E100151 randomized phase III trial [5]. Patients previously treated with an anthracycline, a taxane and trastuzumab were randomized to capecitabine either alone or in combination with lapatinib. TTP was significantly improved in the combination arm (hazard ratio $0.49, \mathrm{p}<$ 0.001 ; median 8.4 months vs. 4.4 months with capecitabine alone). Another strategy after progression on trastuzumab is to continue with trastuzumab but to switch to another chemotherapy regimen. Retrospective data suggested that administration of trastuzumab in multiple treatment lines is beneficial compared with discontinuing trastuzumab at first progression [6-14]. These retrospective data were subsequently confirmed in a prospective randomized trial conducted by the German Breast Group (GBG26) in which patients with progression on trastuzumab-containing therapy received either capecitabine alone or capecitabine combined with trastuzumab beyond disease progression [15]. Compared with capecitabine alone, continuation of trastuzumab was associated with a higher response rate and significantly improved TTP (primary endpoint; hazard ratio 0.69 ; median 8.2 vs. 5.6 months, respectively). Despite these observations, considerable variation exists in the use of trastuzumab both in the first-line setting and after trastuzumab failure. The aim of this retrospective cohort analysis was to describe and understand treatment decision algorithms and to document patient outcomes in Swiss clinical practice.

Swiss Patterns of Care: Trastuzumab beyond Progression

\section{Patients and Methods}

The main objective of the study was to describe therapeutic strategies in Swiss clinical practice in patients with HER2-positive MBC. Physicians from six Swiss breast cancer centers participated in this retrospective cohort analysis. Medical records were reviewed to identify all patients with HER2-positive tumors (immunohistochemistry, IHC, 3+ or fluorescence in situ hybridization, FISH, positive) who received treatment in any line between January 2006 and December 2007, and who experienced progression on trastuzumab-containing therapy. From these records, data on tumor and patient characteristics, HER2 testing method, anti-HER2 therapy, chemotherapy, endocrine therapy, tumor response, duration of therapy, sites of metastasis, disease progression, survival and safety were collected on a case report form and entered into a central database. The data were then analyzed primarily using descriptive statistics. Data collection was finished in December 2009. Comparisons of TTP and overall survival according to chemotherapy regimen were performed using the Mann-Whitney U test and one- and two-sided Monte Carlo tests. Overall survival was calculated from the time of introduction of first non-adjuvant trastuzumab treatment. The Pearson $\chi^{2}$ test was used to compare metastatic sites between the treatment groups.

The study was approved (if required) by the local ethics committee at each of the six participating institutions. The study was also approved by the Swiss Federal Commission of Professional Secrecy in Medical Research.

\section{Results}

\section{Patient Population}

In total, 72 patients were identified for analysis from six Swiss breast cancer centers. All had HER2-positive (IHC3+ or FISH-positive) MBC and had shown disease progression during or following trastuzumab given in the metastatic or relapsed setting. The date of the first breast cancer diagnosis ranged from 1990 to 2007. The median age of patients was 58 years (range 28-81). Two patients had received adjuvant trastuzumab. Nineteen patients had metastatic (stage IV) disease at first diagnosis. Details of tumor biology and prior therapy are shown in table 1.

\section{First-Line Treatment}

The preferred first-line anti-HER2 treatment was trastuzumab in combination with chemotherapy ( $\mathrm{n}=$ $49)$, typically vinorelbine $(n=23)$ or a taxane $(n=22)$. The remaining 23 patients received trastuzumab monotherapy. No significant difference in patient characteristics was detected between the trastuzumab-alone group and the combination group (table 2). However, there was a trend $(\mathrm{p}=0.08)$ towards a higher proportion of patients

Oncology 2011;81:160-166 
Table 1. Summary of baseline characteristics $(\mathrm{n}=72)$

\begin{tabular}{ll}
\hline & Value \\
\hline $\begin{array}{l}\text { Median age, years } \\
\text { Disease status }\end{array}$ & $58(28-81)$ \\
$\quad$ M1 (primary metastatic) & $19(26)$ \\
Hormone receptor status at first diagnosis & \\
$\quad$ Estrogen receptor positive & $40(56)$ \\
Progesterone receptor positive & $21(29)$ \\
Prior treatment before trastuzumab & \\
Neoadjuvant chemotherapy & $5(7)^{\mathrm{a}}$ \\
Adjuvant chemotherapy & $29(40)$ \\
Anthracycline-based & $22(31)^{\mathrm{b}}$ \\
CMF alone & $6(8)$ \\
Other & $1(1)$ \\
Adjuvant trastuzumab & $2(3)$ \\
Adjuvant endocrine therapy & $29(40)$ \\
Prior treatment for advanced disease & \\
$\quad$ (non-trastuzumab) & $24(33)$ \\
$\quad$ Chemotherapy & $14(19)^{\mathrm{c}}$ \\
$\quad$ Endocrine therapy & $20(28)$ \\
\hline
\end{tabular}

Data presented as n (\%) or median (range). CMF = Cyclophosphamide, methotrexate, 5 -fluorouracil.

a Anthracycline-based in 4 of 5 patients, without anti-HER2 therapy in all cases.

${ }^{\mathrm{b}}$ With taxane in 2 patients; with sequential CMF in 4 patients.

${ }^{\mathrm{c}}$ Multiple lines in 5 patients.

Table 2. Patient characteristics before first-line therapy

\begin{tabular}{|c|c|c|c|}
\hline & \multicolumn{2}{|c|}{ Trastuzumab } & \multirow{2}{*}{$\begin{array}{l}\mathrm{p} \\
\text { value }\end{array}$} \\
\hline & $\begin{array}{l}\text { mono- } \\
\text { therapy }\end{array}$ & $\begin{array}{l}\text { with } \\
\text { chemo- } \\
\text { therapy }\end{array}$ & \\
\hline Median age, years & 55 & 59 & - \\
\hline \multicolumn{4}{|l|}{ Number of metastatic sites, $\%$} \\
\hline 1 & 26 & 30 & - \\
\hline$\leq 2$ & 78 & 69 & - \\
\hline \multicolumn{4}{|l|}{ Metastatic sites, $\%$} \\
\hline Liver & 34 & 59 & 0.08 \\
\hline Lung & 34 & 31 & 0.79 \\
\hline Bone & 70 & 55 & 0.31 \\
\hline \multicolumn{4}{|l|}{ Disease status at inclusion } \\
\hline Primary metastatic, $\%$ & 26 & 27 & - \\
\hline Disease-free interval, months ${ }^{\mathrm{a}}$ & 45 & 43 & - \\
\hline
\end{tabular}

Values of p calculated using Pearson's $\chi^{2}$ test.

${ }^{a} \mathrm{n}=53$, excludes patients presenting with metastatic disease.
Table 3. Summary of TTP according to first-line regimen

\begin{tabular}{llll}
\hline & Patients, $\mathrm{n}$ & $\begin{array}{l}\text { Median TTP } \\
\text { months }\end{array}$ & $\mathrm{p}$ value \\
\hline $\begin{array}{l}\text { Trastuzumab monotherapy } \\
\text { Trastuzumab combined }\end{array}$ & 23 & 8.1 & 0.88 \\
$\quad$ with chemotherapy & 49 & 8.1 & \\
$\begin{array}{l}\text { Taxane regimen } \\
\text { Non-taxane regimen }\end{array}$ & 22 & 8.0 & 0.88 \\
\hline \multicolumn{2}{l}{ Values of p calculated with Mann-Whitney U test. } \\
\hline
\end{tabular}

with liver metastases in the combination group (59 vs. $34 \%$ in the monotherapy group) that may have influenced treatment outcome. TTP data according to first-line trastuzumab regimen are shown in table 3. Median TTP was very similar in both treatment cohorts, irrespective of combination partner (no chemotherapy vs. chemotherapy; taxane vs. non-taxane).

\section{Second and Subsequent Treatment Lines}

After progression on the first line of trastuzumabcontaining therapy, 68 of the 72 patients (94\%) received further anti-HER2 therapy. This consisted of trastuzumab in all but 1 patient, who received lapatinib in combination with capecitabine. In the majority of cases, second-line treatment comprised trastuzumab in combination with chemotherapy $(n=51,75 \%)$. As in the first-line setting, the predominant chemotherapy partners were vinorelbine $(n=27)$ or a taxane $(n=16)$. Eight patients receiving second-line chemotherapy with trastuzumab were re-exposed to the same trastuzumab-chemotherapy regimen as they had received in the first line (paclitaxel in 2 patients, docetaxel in 1 patient, vinorelbine in 5 patients). There was no detectable difference in TTP during second-line therapy between patients receiving the same versus another chemotherapy in combination with trastuzumab (median 8.6 vs. 7.1 months, respectively; $\mathrm{p}=0.503$ ). However, the patient numbers in these analyses are too small for meaningful comparison. With further treatment lines, the use of lapatinib combined with capecitabine increased, from $15 \%$ in the third-line setting to $20 \%$ in the sixth line (fig. 1), but it was still selected less frequently than trastuzumab-containing regimens.

The median total duration of anti-HER2 therapy was 20 months (range 0.7-60). After progression on first-line trastuzumab-containing treatment, patients received a median of 4 distinct lines of anti-HER2 therapy (range 


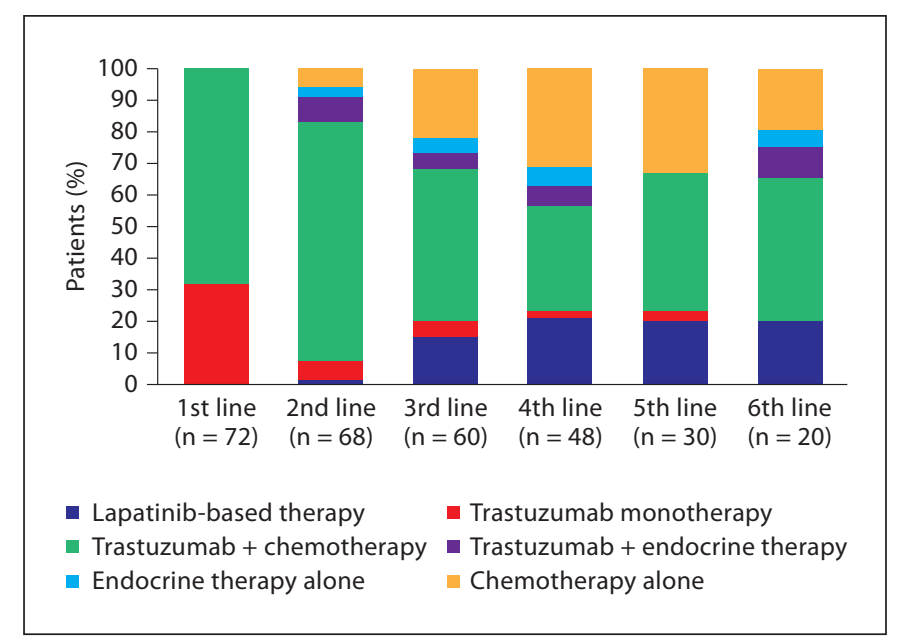

Fig. 1. Summary of treatment according to line.

1-12). With every line of therapy after first administration of trastuzumab, there was a decrease in the median duration of anti-HER 2 therapy and, in most cases, TTP (table 4). There was no apparent correlation between second-line TTP and previous use of trastuzumab as monotherapy or in combination with chemotherapy (median 5.1 months with trastuzumab monotherapy vs. 7.1 months with trastuzumab combined with chemotherapy; $\mathrm{p}=$ 0.92). There was, however, a weak non-significant correlation between TTP in the first- and second-line settings.

Median overall survival in the entire study population was 32.3 months. There was no statistically significant difference in overall survival between those receiving trastuzumab as monotherapy in the first-line setting and those receiving trastuzumab in combination with chemotherapy (median 36.6 vs. 30.0 months, respectively; Mann-Whitney $\mathrm{U}$ test, $\mathrm{p}=0.063$ ).

\section{Brain Metastases}

Overall, 31 of the 72 patients (43\%) developed brain metastases at some point during the study period. Treatments for brain metastases are summarized in table 5 . Median overall survival after diagnosis of brain metastases was 9.8 months (95\% CI 2.8-16.7). Trastuzumab therapy was the predominant treatment immediately after diagnosis of brain metastasis (17 of 31 patients, 55\%). Lapatinib was used in 3 patients (10\%) after diagnosis of brain metastases and in an additional 8 patients with brain metastases in subsequent lines. In total, $68 \%$ of all patients with brain metastasis received at least one anti-HER2 therapy during further lines of therapy.

Swiss Patterns of Care: Trastuzumab beyond Progression
Table 4. Summary of treatment duration and TTP according to line of therapy

\section{Treatment line}

1st 2nd 3rd 4th 5th 6th

Median treatment duration of

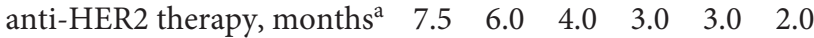

Median duration of $\begin{array}{lllllll}\text { chemotherapy, months } & 3.9 & 4.0 & 3.7 & 3.0 & 3.0 & 2.0\end{array}$

Median time to progression months

${ }^{a}$ Including maintenance treatment.

Table 5. Treatments in patients with brain metastases $(\mathrm{n}=31)$

Systemic therapy immediately after diagnosis of

brain metastases

Trastuzumab

17

Lapatinib $^{\text {a }}$

3

Chemotherapy alone ${ }^{b}$

No systemic therapy

3

No systemic therapy

Anti-HER2-based treatment any time after diagnosis of brain metastases

Lapatinib and trastuzumab (all sequentially) 8

Trastuzumab alone

Lapatinib alone

Neither lapatinib nor trastuzumab Unknown

a 1 patient received trastuzumab in a subsequent line.

${ }^{\mathrm{b}} 1$ patient received lapatinib in a subsequent line.

${ }^{c}$ Including 2 patients for whom additional (sequential) lapatinib use is unknown.

\section{Discussion}

Trastuzumab is considered the standard first-line treatment for HER2-positive MBC and is typically administered in combination with chemotherapy [16]. Our observational study of clinical practice and treatment patterns indicates that in Swiss breast cancer centers, trastuzumab was administered as monotherapy (without chemotherapy) in one third of patients receiving first-line trastuzumab-based treatment for HER2-positive MBC. 
The median TTP with first-line treatment was 8.1 months, both in patients receiving trastuzumab monotherapy and those in whom trastuzumab was combined with chemotherapy. The strategy of first-line trastuzumab monotherapy in selected patients did not appear to negatively influence overall survival. In fact, there were no detectable differences between the cohorts in age, number of metastatic sites before first-line therapy, initial disease status, disease-free interval and institution, although there was a numerically higher proportion of patients with liver metastases in the combination group (59 vs. $34 \%$ in the trastuzumab monotherapy group). This may have influenced outcome, and suggests that patients receiving trastuzumab alone had a better prognosis than those in whom trastuzumab combined with chemotherapy was selected. Consistent with this hypothesis, median TTP in our trial (8 months) is higher than the 4 - to 5 -month median TTP reported in trials of trastuzumab monotherapy in the literature [4].

Results from two small randomized trials comparing trastuzumab monotherapy with the combination of trastuzumab and chemotherapy as first-line treatment contrast in part with our observations. In the HERTAX trial $(\mathrm{n}=99)$, PFS was significantly worse with monotherapy compared with combination therapy (hazard ratio $2.51, \mathrm{p}<0.001$; median 3.9 vs. 9.4 months, respectively) [17]. However, when TTP was measured for the sequence of trastuzumab followed by chemotherapy, efficacy was similar (hazard ratio 1.33, $\mathrm{p}=0.20$; median 9.9 vs. 9.4 months, respectively). There was no statistically significant difference in overall survival between the two groups. In the second trial $(n=107)$, conducted in Japan, the sequence of trastuzumab monotherapy followed by chemotherapy and trastuzumab resulted in similar PFS (to second progression in the sequential arm) to first-line trastuzumab in combination with chemotherapy (median 12.4 vs. 14.6 months, respectively) [18]. However, overall survival was significantly superior for the combination treatment, resulting in premature termination of the trial. As with our study, there are some important caveats when interpreting the results of these two trials. In the HERTAX trial, patients starting with trastuzumab monotherapy were switched to chemotherapy at progression and trastuzumab maintenance therapy was not planned. Furthermore, in both trials there was no central HER2 testing. Consequently, less rigorous patient selection may have led to inappropriate use of trastuzumab monotherapy in some patients. In addition, both trials included relatively small numbers of patients.
In our retrospective cohort study, the proportions of patients receiving taxane or vinorelbine chemotherapy in combination with first-line trastuzumab were similar. There was no significant difference in TTP between these two regimens. This observation is consistent with results of two randomized trials, which showed similar efficacy with taxane or vinorelbine chemotherapy in combination with trastuzumab in the first-line setting $[19,20]$.

Despite the proven efficacy of trastuzumab in MBC, there is still variation in the approach to anti-HER2 therapy, particularly following disease progression on trastuzumab-containing therapy. The anti-HER2 treatment strategy for MBC in Swiss breast cancer centers typically involves multiple trastuzumab-containing regimens. Our results, which are similar to other retrospective studies, indicate that durable disease control can be achieved with repeated or prolonged exposure to trastuzumab. The median TTP of 6 months with second-line treatment in our study is consistent with reports in the literature [21, $22]$. The predominant chemotherapy partners in the second-line setting were vinorelbine and taxane, as in the first-line setting.

Although the combination of lapatinib and capecitabine was approved in Switzerland in May 2007 after trastuzumab failure, and an early access program was available in 2007, the regimen was reserved until the third-line setting in the majority of patients in our study. Furthermore, even in later lines, the use of trastuzumab-based regimens was at least twice as high as that of lapatinibbased regimens. This finding is surprising when considering that data from the randomized E100151 trial of lapatinib and capecitabine were available during the study period, whereas use of trastuzumab beyond progression was supported only by retrospective and anecdotal data at that time. Data from the randomized GBG26 trial suggesting benefit with trastuzumab beyond progression were first presented in December 2007. It is possible that some patients in our study were not eligible for lapatinib, as the regimen was registered only for treatment after exposure to an anthracycline and a taxane. However, this is unlikely to explain why the use of lapatinib was so rare in later lines of therapy, where selection of trastuzumab remained more frequent. A more plausible reason for the preference for trastuzumab over lapatinib concerns practical difficulties with the use of lapatinib. Certainly during the treatment period analyzed in this study, many physicians were more familiar with trastuzumab treatment and were less experienced in the management of lapatinib side effects, and thus may have felt less comfortable with administering lapatinib than trastuzumab. 
The proportion of patients with brain metastases in our study is in line with previous reports and showed a gradual increase with each line of therapy.

In conclusion, in our observational study, trastuzumab monotherapy was chosen in one third of patients in the first-line setting. In this selected patient population, trastuzumab monotherapy appeared to be as effective as trastuzumab in combination with chemotherapy in terms of TTP. However, it remains to be elucidated whether these selected patients may have had an even better outcome if they had received combined first-line immunochemotherapy. The randomized SAKK 22/99 trial has been designed to address this question by comparing trastuzumab alone vs. trastuzumab in combination with chemotherapy as first-line therapy for HER2-positive $\mathrm{MBC}$, and its results are eagerly awaited.

Surprisingly, lapatinib was not used until the thirdline setting; even in later lines, trastuzumab remained the preferred approach and only a minority of patients received lapatinib. The clinical practice adopted in Switzerland between 2006 and 2007, typically involving continued exposure to anti-HER 2 treatment across several lines of chemotherapy, provided sustained disease control and median overall survival of 32 months.

\section{Acknowledgement and Funding}

Support for third-party writing assistance for this work was provided by Roche Pharma AG, Switzerland.

\section{Disclosure Statement}

This work was supported in part by Roche Pharma AG, Switzerland. Study design, data collection, analysis and interpretation of the data, final approval of the manuscript and the decision to submit the paper for publication were the responsibility of the authors and not the sponsors.

Advisory boards: J.H. serves on advisory boards for Roche and GlaxoSmithKline and has received research funding from GlaxoSmithKline. R.v.M. serves on advisory boards for Amgen, Novartis and Roche and has received honoraria from Amgen and Roche. B.T. serves on advisory boards for Roche and GlaxoSmithKline, has received research funding from GlaxoSmithKline and owns stock in Roche. I.W. is an employee of F. Hoffmann-La Roche Ltd. and owns stock in F. Hoffmann-La Roche Ltd. All other authors declare that they have no competing interests.

\section{References}

1 Slamon DJ, Leyland-Jones B, Shak S, Fuchs $\mathrm{H}$, Paton V, Bajamonde A, Fleming T, Eiermann W, Wolter J, Pegram M, Baselga J, Norton L: Use of chemotherapy plus a monoclonal antibody against HER2 for metastatic breast cancer that overexpresses HER2. N Engl J Med 2001;344:783-792.

-2 Marty M, Cognetti F, Maraninchi D, Snyder R, Mauriac L, Tubiana-Hulin M, Chan S, Grimes D, Antón A, Lluch A, Kennedy J, O'Byrne K, Conte P, Green M, Ward C, Mayne K, Extra JM: Randomized phase II trial of the efficacy and safety of trastuzumab combined with docetaxel in patients with human epidermal growth factor receptor 2-positive metastatic breast cancer administered as first-line treatment: the M77001 study group. J Clin Oncol 2005;23:42654274.

- 3 Kaufman B, Mackey JR, Clemens MR, Bapsy PP, Vaid A, Wardley A, Tjulandin S, Jahn M, Lehle M, Feyereislova A, Révil C, Jones A: Trastuzumab plus anastrozole versus anastrozole alone for the treatment of postmenopausal women with human epidermal growth factor receptor 2-positive, hormone receptorpositive metastatic breast cancer: results from the randomized phase III TAnDEM study. J Clin Oncol 2009;27:5529-5537.
4 Vogel CL, Cobleigh MA, Tripathy D, Gutheil JC, Harris LN, Fehrenbacher L, Slamon DJ, Murphy M, Novotny WF, Burchmore M, Shak S, Stewart SJ, Press M: Efficacy and safety of trastuzumab as a single agent in first-line treatment of HER2-overexpressing metastatic breast cancer. J Clin Oncol 2002; 20:719-726.

5 Geyer CE, Forster J, Lindquist D, Chan S, Romieu CG, Pienkowski T, Jagiello-Gruszfeld A, Crown J, Chan A, Kaufman B, Skarlos D, Campone M, Davidson N, Berger M, Oliva C, Rubin SD, Stein S, Cameron D: Lapatinib plus capecitabine for HER2-positive advanced breast cancer. N Engl J Med 2006; 355:2733-2743.

-6 Fabi A, Metro G, Ferretti G, Giannarelli D, Di Cosimo S, Papaldo P, Mottolese M, Carlini P, Felici A, Russillo M, Cognetti F: Do HER-2 positive metastatic breast cancer $\mathrm{pa}$ tients benefit from the use of trastuzumab beyond disease progression? A mono-institutional experience and systematic review of observational studies. Breast 2008;17:499505.

7 Bartsch R, Wenzel C, Hussian D, Pluschnig U, Sevelda U, Koestler W, Altorjai G, Locker GJ, Mader R, Zielinski CC, Steer GG: Analysis of trastuzumab and chemotherapy in ad- vanced breast cancer after the failure of at least one earlier combination: an observational study. BMC Cancer 2006;6:63.

8 García-Sáenz JA, Martín M, Puente J, LópezTarruella S, Casado A, Moreno F, Grande E, Díaz-Rubio E: Trastuzumab associated with successive cytotoxic therapies beyond disease progression in metastatic breast cancer. Clin Breast Cancer 2005;6:325-329.

9 Stemmler H-J, Kahlert S, Siekiera W, Untch M, Heinrich B, Heinemann V: Prolonged survival of patients receiving trastuzumab beyond disease progression for HER2 overexpressing metastatic breast cancer (MBC). Onkologie 2005;28:582-586.

10 Gelmon KA, Mackey J, Verma S, Gertler SZ, Bangemann N, Klimo P, Schneeweiss A, Bremer K, Soulieres D, Tonkin K, Bell R, Heinrich B, Grenier D, Dias R: Use of trastuzumab beyond disease progression: observations from a retrospective review of case histories. Clin Breast Cancer 2004;5: 52-58.

11 Tripathy D, Slamon DJ, Cobleigh M, Arnold A, Saleh M, Mortimer JE, Murphy M, Stewart SJ: Safety of treatment of metastatic breast cancer with trastuzumab beyond disease progression. J Clin Oncol 2004;22: 1063-1070. 
-12 Fountzilas G, Razis E, Tsavdaridis D, Karina M, Labropoulos S, Christodoulou C, Mavroudis D, Gogas H, Georgoulias V, Skarlos D: Continuation of trastuzumab beyond disease progression is feasible and safe in patients with metastatic breast cancer: a retrospective analysis of 80 cases by the Hellenic Cooperative Oncology Group. Clin Breast Cancer 2003;4:120-125.

-13 Cancello G, Montagna E, D’Agostino D, Giuliano M, Giordano A, Di Lorenzo G, Plaitano M, De Placido S, De Laurentiis M: Continuing trastuzumab beyond disease progression: outcomes analysis in patients with metastatic breast cancer. Breast Cancer Res 2008;10:R60.

14 Jackisch C, Eustermann H, Schoenegg W, Soelling U, Stauch M, Goehler T, Kuehn W, Krieger G, Reichert D: Routine clinical usage of trastuzumab (Herceptin) in advanced breast cancer in Germany from 2001 to 2006 (abstract). Breast Cancer Res Treat 2007;4059.

15 von Minckwitz G, du Bois A, Schmidt M, Maass N, Cufer T, de Jongh FE, Maartense E, Zielinski C, Kaufmann M, Bauer W, Baumann KH, Clemens MR, Duerr R, Uleer C, Andersson M, Stein RC, Nekljudova V, Loibl S: Trastuzumab beyond progression in human epidermal growth factor receptor 2-positive advanced breast cancer: a German Breast Group 26/Breast International Group 03-05 study. J Clin Oncol 2009;27:1999-2006.
16 Cardoso F, Senkus-Konefka E, Fallowfield L, Costa A, Castiglione M; ESMO Guidelines Working Group: Locally recurrent or metastatic breast cancer: ESMO clinical practice guidelines for diagnosis, treatment and follow-up. Ann Oncol 2010;21(suppl 5):v15v19.

17 Hamberg P, Bos M, Braun HJJ, Stouthard JML, van Deijk GA, Erdkamp FLG, van der Stelt-Frissen IN, Bontenbal M, Creemers GJM, Portielje JEA, Pruijt JFM, Loosveld OJL, Smit WM, Muller EW, Schmitz PIM, Seynaeve C, Klijn JGM: Randomized phase II study comparing efficacy and safety of combination-therapy trastuzumab and docetaxel vs. sequential therapy of trastuzumab followed by docetaxel alone at progression as first-line chemotherapy in patients with HER2+ metastatic breast cancer: HERTAX trial. Clin Breast Cancer 2011;11: 103-113.

18 Inoue K, Nakagami K, Mizutani M, Hozumi Y, Fujiwara Y, Masuda N, Tsukamoto F, Saito M, Miura S, Eguchi K, Shinkai T, Ando M, Watanabe T, Masuda N, Ohashi Y, Sano M, Noguchi S: Randomized phase III trial of trastuzumab monotherapy followed by trastuzumab plus docetaxel versus trastuzumab plus docetaxel as first-line therapy in patients with HER2-positive metastatic breast cancer: the JO17360 Trial Group. Breast Cancer Res Treat 2010;119:127-136.
19 Burstein HJ, Keshaviah A, Baron AD, Hart RD, Lambert-Falls R, Marcom PK, Gelman $\mathrm{R}$, Winer EP: Trastuzumab plus vinorelbine or taxane chemotherapy for HER2-overexpressing metastatic breast cancer: the trastuzumab and vinorelbine or taxane study. Cancer 2007;110:965-972.

20 Andersson M, Lidbrink E, Bjerre K, Wist E, Enevoldsen K, Jensen AB, Karlsson P, Tange UB, Sørensen PG, Møller S, Bergh J, Langkjer ST: Phase III randomized study comparing docetaxel plus trastuzumab with vinorelbine plus trastuzumab as first-line therapy of metastatic or locally advanced human epidermal growth factor receptor 2-positive breast cancer: the HERNATA study. J Clin Oncol 2011;29:264-271.

-21 Metro G, Giannarelli D, Gemma D, Lanzetta G, Ciccarese M, Papaldo P, Gamucci T, Lorusso V, Mottolese M, Magnolfi E, Cognetti F, Fabi A: Time to first tumor progression as outcome predictor of a second trastuzumabbased therapy beyond progression in HER-2 positive metastatic breast cancer. Breast J 2010;16:66-72.

22 Bartsch R, De Vries C, Pluschnig U, Dubsky P, Bago-Horvath Z, Gampenrieder SP, Rudas M, Mader RM, Rottenfusser A, Wiltschke C, Gnant M, Zielinski CC, Steger GG: Predicting for activity of second-line trastuzumabbased therapy in HER2-positive advanced breast cancer. BMC Cancer 2009;9:367. 